\title{
Key skills by design: adapting a central Web resource to departmental contexts
}

\author{
Claire McAvinia \\ University College London \\ email: c.mcavinia@ud.ac.uk
}

Web-based delivery of support materials for students has proved to be a popular way of helping small teams to implement key skills policies within universities. The development of 'key' or 'transferable' skills is now encouraged throughout education, but resources (both in terms of staffing and budget) tend to be limited. It is difficult for key skills teams to see learners face to face, and not feasible to print or distribute large amounts of paper-based material. Web-based delivery presents a means of overcoming these problems but it can result in generic study skills material simply being published online without due consideration of the needs of different groups of learners within different subject disciplines. Therefore, although a centralized Website for skills provision can overcome logistical problems, it may be perceived as irrelevant or unusable by the student population. This paper presents a model for Web-based delivery of support for key skills which incorporates two separate approaches to the design of these resources. The model was implemented as part of a wider key skills pilot project at University College London, over a period of one year. It includes a 'core' Website, containing information and resources for staff and students. These can also be accessed via customized, departmental key skills homepages. This paper presents the basis for the design choices made in preparing these materials, and the evaluation of some of the pilot departments using them. It then draws some wider conclusions about the effectiveness of this design for supporting skills development.

\section{Introduction: the key skills agenda}

Key skills are referred to increasingly by government and employers in the UK as being essential to ensuring long-term economic prosperity and an adaptable workforce. The new prioritization of skills at government level is reflected in policy statements and strategy at 
all levels of education and training (DfES, 2001), and indeed by the renaming of the Department for Education and Employment as the Department for Education and Skills.

Within higher education, key skills are represented as 'general' skills which employers expect graduates to bring to the workplace in addition to the specialist knowledge they have acquired in the course of their degree studies (Atkins, 1999). They are usually defined as being separate from discipline-specific skills and assumed to be easily 'transferable' from one context to another. Although there is a continuing debate about the nature of these skills, whether they can be transferred, and whether employers are in fact able to predict what kinds of skills they will need from future graduate cohorts (Drew, 1998), the agenda has been steadily advanced by HE policy in recent years.

Key skills have been grouped under a variety of labels: 'core', 'personal transferable' and 'generic' to name a few (Bennett, Dunne and Carré, 1999). Attempts have also been made on a national level to draw up a definitive list of key skills. The Qualifications and Curriculum Authority (QCA, 2000) has undertaken this for pre-university and National Vocational Qualifications, while Dearing (DfEE/NCIHE, 1997) nominated four key skills which he recommended should be developed in the course of a student's higher education: communication, numeracy, information technology and learning to learn.

There remain many differences between the institutions' and subject disciplines' definitions of key skills. Institutions may define key skills in terms of 'graduateness', and the attributes they would like their graduates to have. For subject disciplines, the various categorizations of skills may stem from the difficulties in defining which skills are subjectspecific and which are 'general' or transferable (Bennett, Dunne and Carré, 2000). This is reflected in the extensive lists of skills which have emerged from the subject benchmarking process instigated by the Quality Assurance Agency for Higher Education (QAA, 2001). However, although the terminology and the lists themselves are not universally agreed, most will include communication, improving own learning, information technology skills, numeracy, teamwork and problem-solving.

\section{Key skills delivery in higher education}

\section{Institutional approaches}

Delivering provision for key skills to students in higher education is made more difficult by the fact that there is a wider debate about whether it is the role of higher education to prepare graduates for life after their studies and for the workplace (Drew, 1998). If we accept that this is a significant responsibility of the university, then to what extent should it be resourced and should provision be made within the curriculum? A second issue here is that of support for any initiative once it is under way: university management may be reluctant to invest in long-term provision, the effects of which are insufficiently documented (Hesketh, 2000), and towards which greater and lesser amounts of government interest are directed at various times (Drew, 1998).

As a consequence of these debates, there is an extensive range of approaches to the implementation of key skills from one university to another (Fallows and Steven, 2000). Some universities undertake institution-wide audits for key skills and set firm targets for skills delivery and assessment. Others may choose a project-based approach, whereby exploratory work is done with small groups of academic staff and a range of methods 
piloted. However, almost all of these approaches rely on centralized provision for skills development: this will either be in the form of centrally delivered, optional support for all students, or as self-contained generic units developed by a central team and 'bolted on' to individual subject curricula.

\section{Practical issues for delivery}

The combined effects of the issues discussed above impact strongly on the practical delivery of key skills support to university students. Day-to-day responsibility for provision tends to lie with a small centrally-located team which must address the needs of all students. Sometimes this team may be part of an existing service division, in which case it has the benefit of that support structure, but it may also be a project team working in isolation. Centrally delivered skills-related activities (whether optional and isolated from subject delivery or 'bolted on') may be well-intentioned but perceived as irrelevant by the students themselves.

Budget restraints mean that the printing and distributing of large quantities of paperbased material are not usually possible, or can only be done at critical times (for example, in the first term for first-year undergraduates). The nature of the support material - linking as it does to study skills, problem-solving and even revision techniques - means that it might be required quickly by students at any time. A small team would probably find it impossible to meet this need on a face-to-face basis, and so the Web seems to present an ideal means of overcoming several difficulties: materials can be made available to students via a centralized Website, and these will be available at all times and to all students with access to the Internet. However, it raises a number of further issues, which will now be discussed.

\section{Designing Web-based resources for key skills}

A central university Website for key skills will often form the baseline support for students' skills development, and many excellent generic Websites have been produced to cover a variety of skills, both by teams in the UK and internationally. ${ }^{1}$ The resources found on these sites are wide-ranging and include study skills guidelines and materials, Weblinks, CAL packages, references to paper-based information or books, and opportunities for skills development in extra-curricular activities (such as student tutoring, student union activities or part-time work).

The teams producing these kinds of sites may already have compiled large amounts of paper-based support materials or produced their own, based on the needs of their particular students. Although there are some examples of where these have simply been made available online as heavily text-based Webpages (perhaps due to staffing or resource constraints), in most cases they have been adapted for publication on the Web.

This kind of adaptation is important for usability, and it draws on elements of good practice in Web design. Guidelines for Web usability (for example, Nielsen, 2000) centre on ease of use. Nielsen focuses particularly on fast access to Web-based material: users should be given the easiest and quickest routes to the information they require. Text should be 'chunked' into small sections so that it is easily scannable by the reader, and long documents divided logically into separate Webpages which can then be interlinked. External Weblinks should be selected carefully in terms of their relevance to the user. 
These design considerations are highlighted here because they are important in the context of key skills: learners may need to use this support as they try to complete assignments or job applications, or simply because they have a limited amount of time to give to skills development where it is taking place outside the curriculum.

However, the design of an effective generic resource for key skills can also draw on instructional design approaches for the preparation of open and distance learning materials. The learners using a central key skills Website are at a distance from the team, if not actually categorized as 'distance learners' by the university. Although they may need to find information quickly for one circumstance, they may also seek an opportunity to develop or learn a particular skill in more depth. Key skills teams will normally have few opportunities to meet with the students for whom they design provision, but these overlapping requirements in terms of information retrieval and learning objectives must be addressed by the resources they produce.

Whereas Nielsen (2000) advocates design for quick and straightforward information retrieval, Rowntree (1994) considers the learners' needs and provides a set of checklists for each stage of the preparation of materials. He discusses specific choices for the writer/designer: the medium, which learning objectives will be met by which materials, the management of text and the tone of the text, appropriate use of illustrations, and clear guidance around the materials for the learner. Although his suggestions reflect some of the considerations for a well-designed Website, there is one important difference and it is a difference which arguably needs much greater emphasis in the context of designing Webbased resources for key skills: the materials should show an 'obvious awareness of different learners' needs' (Rowntree, 1994).

A key skills team is likely to have or to obtain some data on the student population, such as the distribution of age groups and home/overseas students between various departments, and technical information about how the students will access the Web-based resources. All of this will help with the design of a generic central Website, but a detailed investigation of the characteristics and needs of the learners raises the issue of different cultures within the university itself. Although many key skills teams include members of staff who have taught (sometimes as lecturers within the same university) or worked with students over a period of time, this 'obvious awareness' may not be there for specific subject groups. In order to provide a useful Web-based resource, the team may need to progress from Nielsen's (2000) view of well-organized, speedily available information, to Rowntree's (1994) model of a distance-learning resource designed to meet specific needs, and to be studied over a period of time.

\section{Designing for different contexts}

If a Web-based resource for key skills is truly to reflect the needs of its users, then it needs to take into account the cultures of the departments in which those learners are located. Becher (1989) finds that subject disciplines function in different ways, and that staff identify with the subject community rather than with their respective institutions. This suggests that a 'contextualized' approach to supporting key skills may be more effective than a heavily centralized one. If we treat departments as cultures and design for each in a different way, we are contextualizing our approach to supporting learning. This is 
supported by theories of situated learning, but links also to participatory design, which seeks to include the users in the design and implementation of new technologies (Schuler and Namioka, 1993).

By taking the situated learning and participatory design approaches into account, we might arguably be able to produce materials with greater perceived 'relevance' to the needs of the learners. McLoughlin and Oliver (2000) have applied theories of situated learning and communities of practice to the field of instructional design. They argue: 'One of the limitations in current instructional design models is that they do not fully contextualize the learning experience, and are themselves the products of particular cultures.' They make a distinction between local sites, designed for one context and culture, and international sites that reach across many cultures. Their use of local and international is not dependent on geographical locations, but rather on culture. In their case, the particular culture is the indigenous Australian population, but their work suggests that it may be important to differentiate between subject cultures too. The reasons to design for an ethnic group, or for non-traditional or part-time learners may be much clearer than the reasons to design differently for particular subject disciplines. In designing a model for Web-based skills resources at UCL, it was decided to try to establish whether contextualizing the resources for particular departments would make a difference to how the materials were received and used.

\section{The UCL model}

The UCL key skills Website was developed to support a wider key skills pilot, which ran during the academic session 2000-1. This pilot stemmed from an initial audit of their curricula for key skills by departments, and the subsequent compilation of a list of skills which tutors felt were essential for their students to have on graduation. A paper-based

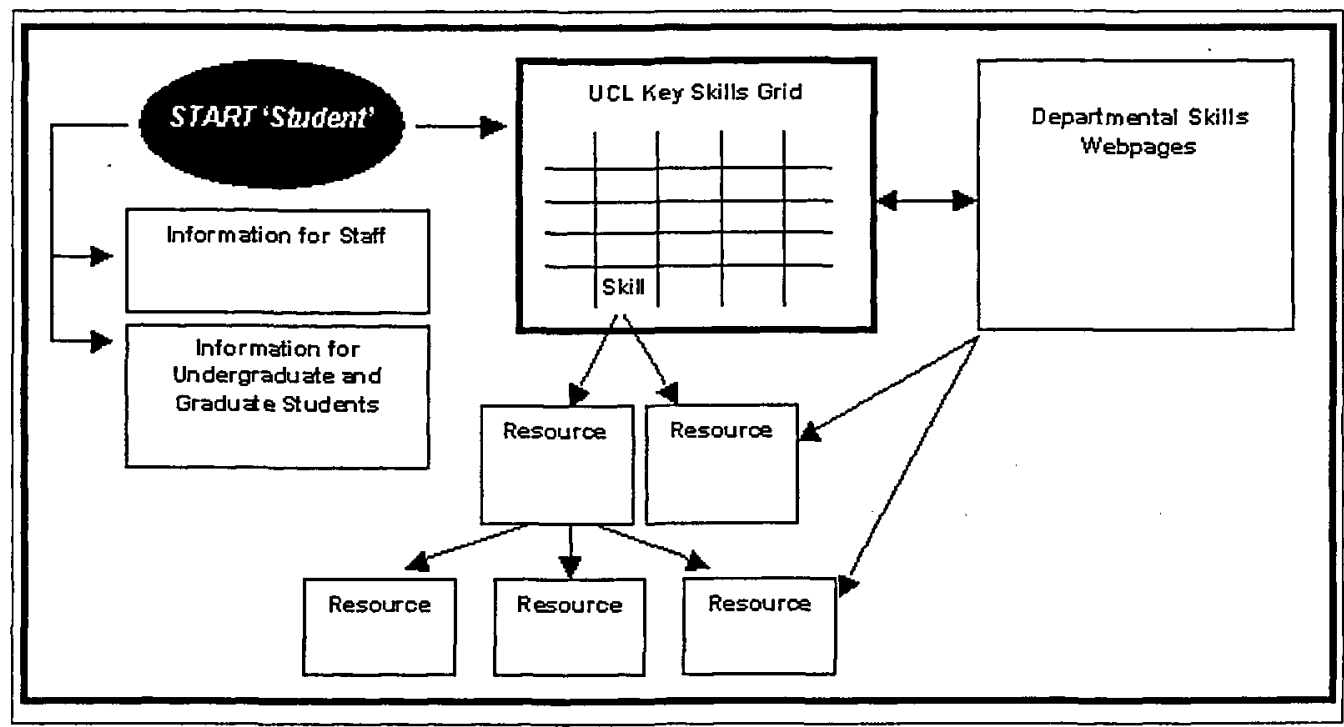

Figure I: Model of the UCL key skills Website 
profile was distributed to first-year undergraduate students from six departments, asking them to assess their own skills and to identify those needing further development. They were then encouraged to use the Website to support this process.

The design of the Website (shown at Figure 1) includes two approaches to the delivery of the resources: a centralized site for key skills and also customized Webpages for departments. As can be seen from the diagram, the customized pages draw on the resources at the central site, but provide a different 'front end' from these: this has the aim of 'personalizing' and familiarizing the resource to enhance usability, so that the student can find his/her way around the materials without having to use the central 'anonymous' skills page.

This was not a case of simply 'rebranding' the site with the department's house style for its own Webpages: rather, it was designed to allow departments to prioritize certain skills at certain times in a course, and to allow students to take a particular route into the resources via a course topic or type of assignment. A number of wider potential benefits were also intended: the departmental skills pages might give ownership of skills development back to departments without adding to the workload of the academic staff from those departments, and potentially help skills development to become integrated as part of the department's provision.

In terms of design, the two means of accessing the support material (generic and subjectspecific) reflect aspects of the two models discussed above: Nielsen's (2000) idea of a clearly organized, 'just-in-time' resource is important in the design of the central Website. Rowntree's (1994) guidance for the design of distance-learning resources was also considered, but arguably to a greater extent in the case of the customized, subject-specific sites tailored to particular objectives. However, a variety of models might be called on in addressing the needs of different subject disciplines: McLoughlin and Oliver's (2000) course design principles for 'culturally inclusive instructional design' suggest that a range of perspectives may have to be accommodated by the design of resources. This is important in avoiding a scenario where one culture appears to present another with a 'deficit model', that is, where a central team or potentially even a course team from a subject discipline may present one design of the resources as the ideal.

McLoughlin and Oliver (2000) refer to creating access to a variety of resources in order to ensure multiple perspectives: the tailored departmental pages provide an opportunity to pool supporting resources, be they subject-specific (for an assignment), generic (for the study skills or other skills used in completing that assignment), or a combination of both. In many discipline areas there are supporting subject-specific skills materials available. A customized Webpage provides tutors with an opportunity to highlight these, and they can bridge the gap between generic and discipline-specific resources.

A further issue relating to content arises in the context of the language and terminology of key skills. Skills lists, and the materials designed to support the development of skills, may need to be adapted to the language of a subject discipline. The definition of a particular skill written by the key skills team may have quite a different meaning within a subject discipline, and the members of that discipline may perceive it as reductive. Alternatively, such a definition may be irrelevant to the subject discipline where that skill encompasses the whole subject area. One example might be that of numeracy, where a key skills definition would essentially be meaningless to the members of a mathematics department. 
Therefore, in approaching particular departments to discuss the customization of our resources for their students, we encouraged them not only to consider the design, organization and content of the Webpages, but to define what they meant by each of the skills they hoped the sites would support. They were also asked to look for opportunities to provide authentic ways of using the key skills materials within the coursework, and although this proved difficult it yielded some positive results which are now discussed.

\section{Examples of contextualized resources for key skills}

This section discusses the preparation of two sets of customized key skills pages: one each for the departments of Geography and Biology at UCL. Both sets of pages were produced as part of the key skills pilot discussed above.

\section{Department of Geography}

For this department, the format and structure of the customized pages was discussed with academic tutors and an academic administrator. The pages were then designed in their departmental 'house' style but they were stored with the key skills Website during the pilot phase. Figure 2 shows the customized key skills home page for Geography, and Figure 3 the key skills homepage which conforms to the central UCL Web design.

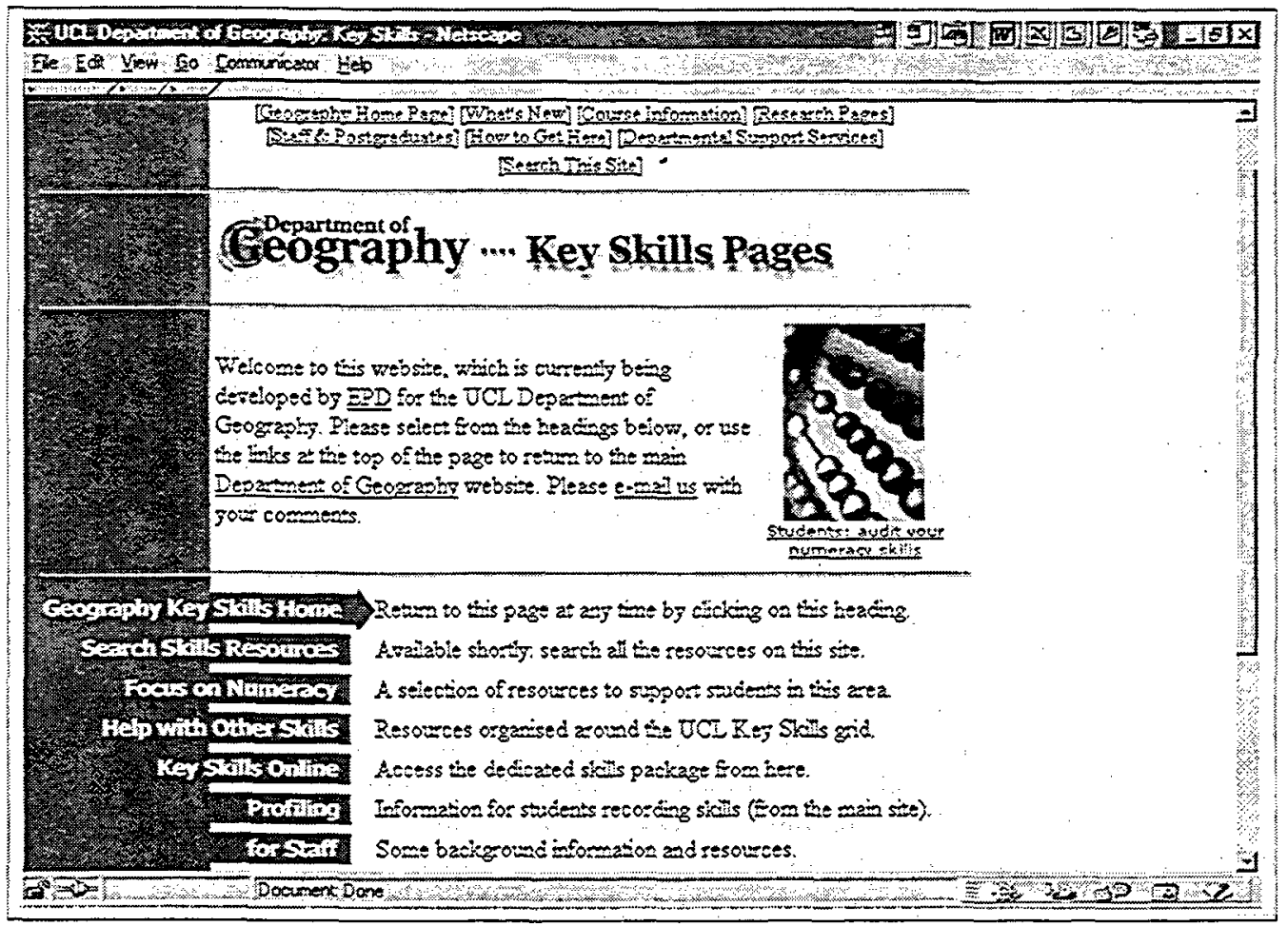

Figure 2: The Geogrophy key skills homepoge 


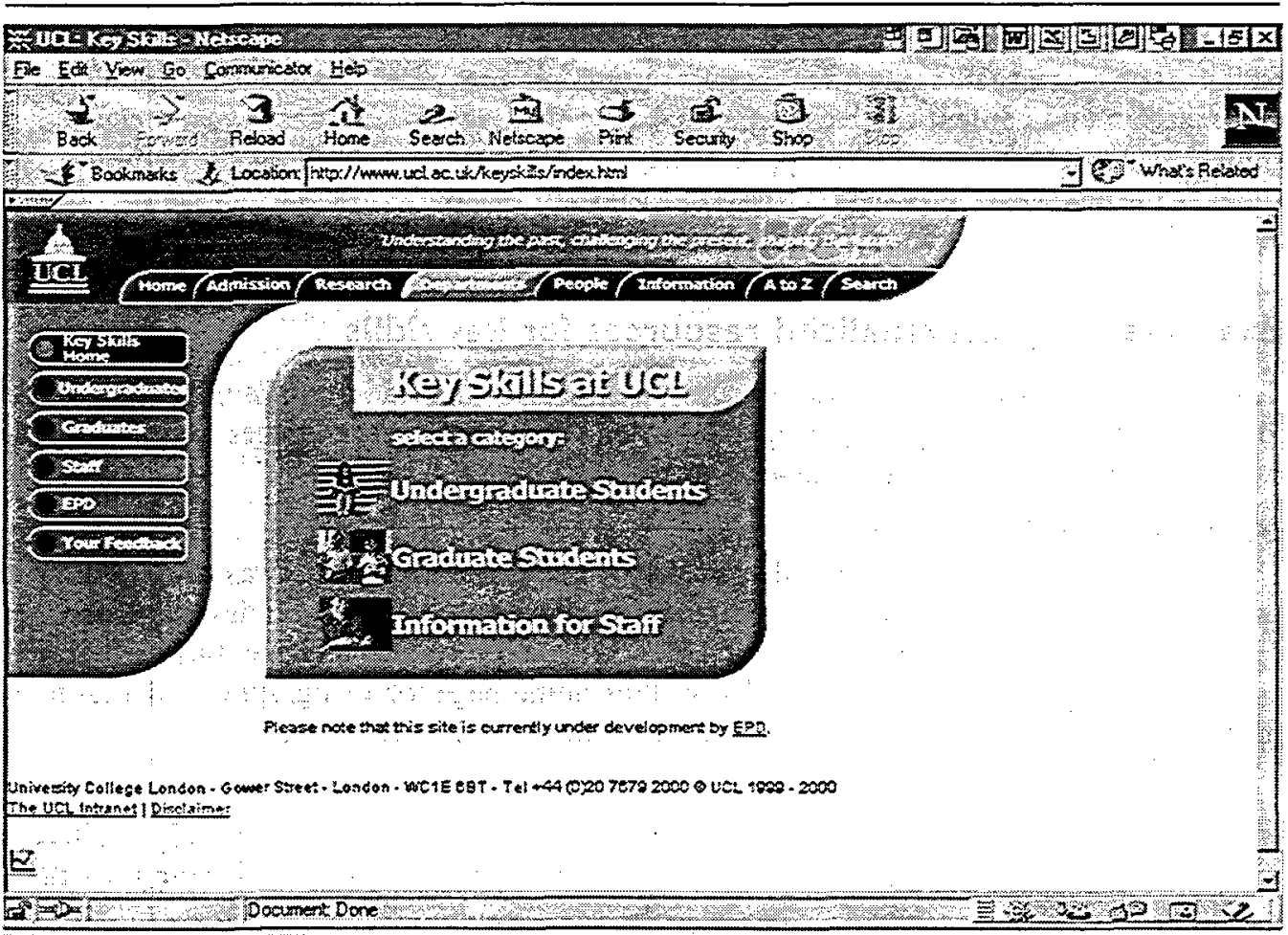

Figure 3: The main key skills homepage

Mentioned previously was the idea that the customized pages could be used by departments to prioritize particular skills at particular times. In this case, the department wished to focus on numeracy skills for one of their undergraduate courses. They felt that resources which would help students to revise some of their skills, and then lead into more complex skills such as data analysis, would be of help in supporting this course. The selection of appropriate resources to be included depended on discussion about what 'numeracy' meant for these courses and these students. The links included quality generic resources as well as the staff homepages of the course coordinator, and a range of material he had gathered or noted, but had not previously been able to present to the students in the context of supporting their coursework.

The students were asked to use the site to prepare for a student-led session, and subsequently reported informally that it had been useful for this class. Formal evaluation from a questionnaire, distributed to the year group as a whole, indicated greater usage of this site and the central site by these students than for students across five other key skills pilot departments. Although this finding is based on questionnaires distributed to a relatively small group (25 students), it suggests that the inclusion of the contextualized resources within course activity had a positive impact on the learners. The tutors' feedback from the department suggested that they were giving further thought to the ways in which Web-based resources might be used in this area, and it seems likely that the department will endeavour to continue this work independently. 


\section{Department of Biology}

Customized pages were designed for the Department of Biology in the latter half of the pilot phase, when the Web development work was almost complete. These are being accessed by students from that department in the current academic session, and as a consequence, there is no formal evaluative data available. This case differed slightly from that of the Geography Department, in that there was no obvious course selected for which the pages could immediately be used as support. This highlights a difficulty in key skills delivery: tutors may have no previous experience of explicitly describing and supporting skills in their curricula, and therefore may be unsure of where such support can best be given.

In this case, the tutor involved suggested that the work might be based on an existing audit of key skills within the courses offered at undergraduate level by the department. The department had produced a 'map' of key skills in the undergraduate curricula and represented this by means of a paper-based diagram of skills gained in each unit of the course. The paper-based diagram was converted to an 'image map' (shown at Figure 4) around which the key skills resources were organized. Students using the map online are directed from a course unit to details of where the key skills will be gained in that particular unit, with these in turn being mapped to supporting materials.

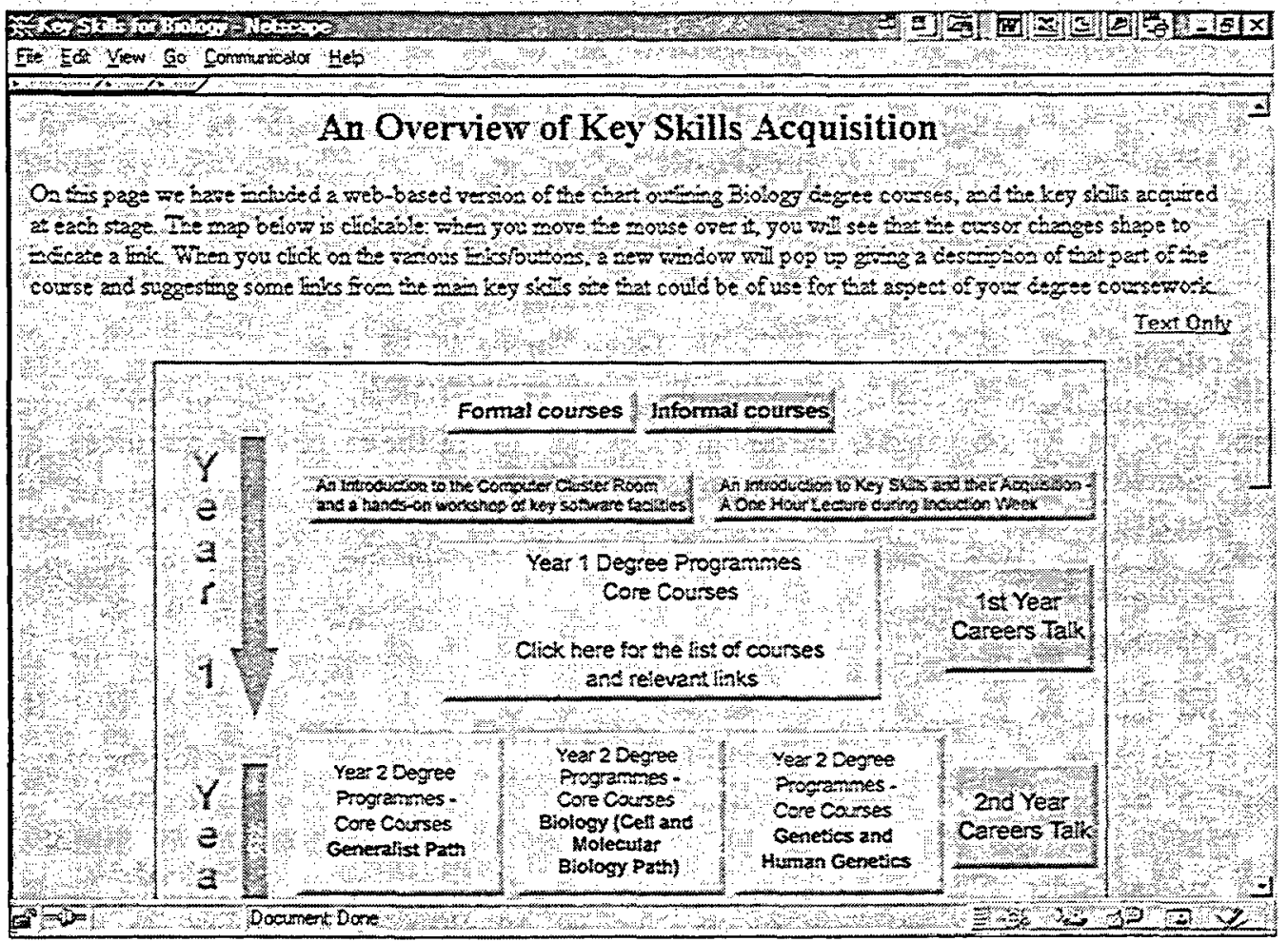

Figure 4: The Biology key skills image map 
The department has expressed a positive response to the resource and it provides an interesting model in terms of the organization of the undergraduate curriculum around key skills. However, although it adopts the approach of customizing resources, it does not include them as part of course activity. Without evaluative data it is impossible to assess whether this approach will be more or less successful than that adopted for the Department of Geography, and further evaluation with the tutors and students concerned is required.

\section{Lessons from the pilot}

\section{Design considerations}

This pilot work involved two approaches to the design and delivery of Web-based key skills resources: the central resource and the customized, individual sets of departmental pages. It has demonstrated that a central resource provided in tandem with tailored, customized resources for departments may be more effective in delivering support for skills development to undergraduate students than the purely centralized or 'bolt-on' provision of generic materials. The design of the central resource may benefit from a consideration of the principles of designing for distance learning, as well as those of good Website design, although here it was difficult to gather large amounts of data to support this view.

The adaptation of the resources to particular subject areas is not simply a question of changing the packaging or 'rebranding' them. It requires a meaningful dialogue with members of the department about the customization of the pages, and could even be considered in terms of a participatory approach. It is important to find and include authentic, contextually relevant material on these pages as well as links to the generic skills development material, and perhaps most important is to encourage tutors to find opportunities to incorporate the material within course activity. Without these opportunities the customized resources risk having to be 'bolted on' as generic material has been in the past (Fallows and Steven, 2000).

\section{Practical considerations}

Work on the UCL key skills site was undertaken by a Web developer (the author) whose time was exclusively devoted to the design and population of the central Web resource and, over time, the customized key skills pages for departments. The outcomes of the pilot suggest that the approach has been effective, but in turn imply that this kind of approach needs a dedicated Web developer on the key skills team. The location of the Web developer within the team may also be beneficial in terms of awareness of the issues in key skills, skills terminology and the range of existing generic resources.

\section{Future development}

The final phase of the pilot envisaged the departments taking responsibility for the pages after the initial pilot phase ended. This process is not complete at UCL, and the transfer is not something we have pressurized the pilot departments involved in this work to do. Rather, they have been encouraged to think about who would be best placed to take responsibility for the pages and to expand the resources. Not every department will have a dedicated Web developer, and in some departments Websites are maintained centrally or by an academic who already has a large number of other responsibilities. Therefore, the transfer may not be something that can be done quickly. 


\section{Conclusions}

In spite of the varying extent to which skills policies have been implemented in HE, and the many issues they raise, the evidence of the potential benefits to students of developing their key skills are now becoming clearer (Fallows and Steven, 2000). Students are being encouraged to reflect on their learning, to take responsibility for it and to formulate their own objectives for university and life afterwards. They are also learning a language to describe their abilities and to record their achievements, and are effectively laying the foundations for their lifelong learning.

Although the delivery of skills support to students is problematic, patterns as to how it can best be done are starting to emerge. Drew, Shaw and Mowthorpe (2000) suggest a continuum whereby generic key skills materials and activities are gradually integrated into coursework, and they document the increased usage of the resources by students where this has been done. This would seem to support the model presented here: a well-designed, generic Web-based resource may be an effective (and cost-effective) form of baseline provision, but the benefits to students may be greater by adapting and eventually including such material within course activity.

The short timescale of the project described here means that further research would be necessary to determine its long-term impact, and to gather additional evaluative data from the tutors and students involved. However, it does perhaps demonstrate that the nature of key skills support is evolving: if such support is to rely on the Web as its keystone, then a 'decentralizing' approach may have to be built in to planning and budgeting processes. One of the most recent surveys of key skills development as a whole in higher education includes a number of accounts of where face-to-face skills development is now taking place with individual departments, and is considered to be essential in taking the initiative forward (Fallows and Steven, 2000). It may well be the case that Web-based support will follow suit.

A related issue is that of responsibility for the design and development of the Website(s) for a key skills team. If this work is done by a member of the team, then perhaps that role could increasingly be described as that of a learning technologist or 'new specialist' (Beetham, 2001). This may have implications for the needs of such team members in terms of staff development and future responsibilities. Where Web development work is carried out centrally, there may be a need to examine the resource available for this if support is to be tailored to specific departments. The likelihood of future initiatives needing the same, or greater, degrees of technical support might also have to be examined by the institution. One example of this appears in the related initiative of student profiling, which has the potential to make heavy demands on a range of systems (including virtual learning environments and student records systems) once implemented online.

The crossover between learning technologies and Web-based support for key skills is again highlighted by the final report of the continuation project for Key to Key Skills (Drew, Catto and Anderson, 2001). In one institution the integration of the project's Key Skills Online package was the catalyst for the institution to begin formulating a policy on online learning. In another case, the implementation of the package was a trigger to frame their key skills policy. Therefore, it seems likely that the design of this kind of provision will continue to change and grow along with the technologies themselves. 


\section{Notes}

1 Many examples have been included as hyperlinks from the UCL key skills site: http://www.ucl.ac.uk/keyskills/grid.html

\section{References}

Atkins, M. (1999), 'Oven-ready and self-basting: taking stock of employability skills', Teaching in Higher Education, 4 (2), 267-80.

Becher, T. (1989), Academic Tribes and Territories: Intellectual Enquiry and the Culture of the Disciplines, SRHE/Open University Press.

Beetham, H. (2001), Career Development of Learning Technology Staff: Scoping Study Final Report, http://www.sh.plymouth.ac.ukleds/effects/jcalt-projectl

Bennett, N., Dunne, E. and Carré, C. (1999), 'Patterns of core and generic skill provision in higher education', Higher Education, 37, 71-93.

Bennett, N., Dunne, E. and Carré, C. (2000), Skills Development in Higher Education and Employment, Milton Keynes: Open University Press.

DfEE/NCIHE (1997), Report of the National Committee of Enquiry into Higher Education: Higher Education in the Learning Society, Recommendation, 23. Sheffield: Crown Copyright.

DfES (2001), Education and Skills: Delivering Results, A Strategy to 2006, Suffolk: Crown Copyright.

Drew, S. (1998), Key Skills in Higher Education: Background and Rationale, SEDA Special no. 6, Birmingham: SEDA Publications.

Drew, S., Shaw, M. and Mowthorpe, D. (2000), Key to Key Skills Project Final Report, http://www.shu.ac.uk/keytokey/finalrep4.pdf

Drew, S., Catto, C. and Anderson, V. (2001), TQEF Transferability and Continuation Funding for TLTP3 Key to Key Skills Project: Final Report, http://www.shu.ac.uk/keytokey/ cont_final_rep.htm

Fallows, S. and Steven, C. (eds) (2000), Integrating Key Skills in Higher Education, London: Kogan Page.

Hesketh, A. (2000), 'Recruiting an elite? Employers' perceptions of graduate education and training', Journal of Education and Work, 13 (3), 245-71.

McLoughlin, C. and Oliver, R. (2000), 'Designing learning environments for cultural inclusivity: a case study of indigenous online learning at tertiary level', Australian Journal of Educational Technology, 16 (1), 58-72.

Nielsen, J. (2000), Designing Web Usability: The Practice of Simplicity, Indianapolis: New Riders

QAA (2001), Benchmarking Academic Standards: Benchmarking Statements, http:// www.qaa.ac.uk/crntwork/benchmark/benchmarking.htm 
QCA (2000), National Qualifications: Key Skills Specifications,

http://www.qca.org.uk/nq/ks/main2.asp

Rowntree, D. (1994), Preparing Materials for Open, Distance and Flexible Learning, London: Kogan Page.

- Schuler, D. and Namioka, A. (eds) (1993), Participatory Design: Principles and Practices, Mahwah, NJ: L. Erlbaum Associates. 